\title{
Brain thermal kinetics at brain-eyelid thermal tunnels overcoming COVID-19 thermometry limitations for automated asymptomatic infection detection in concert with physical and biological principles
}

M Marc Abreu ${ }^{1,2}$, Ricardo L Smith ${ }^{3}$, Trevor M Banack ${ }^{1}$, Alexander C Arroyo ${ }^{4}$, Robert F Gochman $^{4}$, Anna L Clebone ${ }^{1}$, Feng Dai ${ }^{5}$, Michael F Bergeron ${ }^{6}$, Ala S Haddadin ${ }^{1}$, Tyler J Silverman ${ }^{1}$, Adriano F Da Silva ${ }^{7}$, and David G Silverman ${ }^{1,8}$

${ }^{1}$ Department of Anesthesiology, Yale University School of Medicine

${ }^{2}$ Department of Ophthalmology and Visual Science, Yale University School of Medicine

${ }^{3}$ Department of Morphology and Genetics, Paulista School of Medicine, Federal University of São Paulo

${ }^{4}$ Department of Pediatric Emergency Medicine, North Shore - Long Island Jewish Medical Center

${ }^{5}$ Yale Center for Analytical Sciences, Yale School of Public Health, Yale University

${ }^{6}$ Department of Physical Therapy, Augusta University Medical Center, Medical College of Georgia, Augusta University

${ }^{7}$ Department of Radiology, Heart Institute, University of São Paulo Medical School

${ }^{8}$ John B. Pierce Foundation Laboratory, Yale University

October 13, 2021

Rich media available at https://player.vimeo.com/video/462269350?h=112c3228b6

Rich media available at https://player.vimeo.com/video/462269319?h=55cd144dfd

Rich media available at https://player . vimeo. com/video/462269295?h=eb25405c2d

\section{Hosted file}

Authorea_BrainThermalKinetics-viaBTT-AsympDtection_TLY_Yale_Abreu-et-al_0CT2021_Submitted-CompF-1.pdf available at https://authorea.com/users/364278/articles/541438-brain-thermal-kinetics-atbrain-eyelid-thermal-tunnels-overcoming-covid-19-thermometry-limitations-for-automatedasymptomatic-infection-detection-in-concert-with-physical-and-biological-principles 